\title{
Optical Simulation of PV Solar Concentrators by two Inverse Characterization Methods
}

\author{
Antonio Parretta ${ }^{1,2, *}$, Francesco Aldegheri ${ }^{1}$, Andrea Antonini ${ }^{3}$, Mariangela Butturi ${ }^{3}$, Paolo Zurru ${ }^{3}$ \\ ${ }^{1}$ Physics Department, University of Ferrara, Ferrara, 44122, Italy \\ ${ }^{2}$ ENEA Centro Ricerche “E. Clementel”, Bologna, 40129, Italy \\ ${ }^{3}$ CPOWER Srl, Marano Vicentino, 36035, Italy
}

\begin{abstract}
The optical characterization of solar concentrators for photovoltaic applications is increasing its importance with the recent first commercialization of so me CPV technologies. In th is paper two effective characterization methodologies are presented, applied to a representative optics of concentration. Two "inverse" methods are compared: the firstly presented inverse illumination method and another approach, derived by the inverse luminescence method, operating with electrolu minescent light. Both use ideal lambertian sources for producing the inverse light path and can be applied to any type of solar concentrator (photovoltaic or thermodynamic). The optical simulations show their equivalence to get the angular distribution of the transmission efficiency function, but the original inverse illumination method demonstrates a simpler configuration and permits a significantly faster simulation process.
\end{abstract}

keywords Solar Concentrator, Nonimaging Optics, Optical Characterization, Modeling and Analysis

\section{Introduction}

The concentration of solar radiation plays a key role in the field of renewable energies, as it can be effectively applied to thermal, thermodynamic, photovoltaic (PV) and even hybrid thermal/photovoltaic technologies[1-10]. In concentrating photovoltaic systems (CPV) the size of the photovoltaic receiver (solar cell) is reduced by a factor equal to the geometric concentration ratio, and this has a strong, positive impact on the cost of the total PV concentrator, opening perspectives for the use of more sophisticated and more efficient devices. The concentrating optics is one specific component of the photovoltaic concentrator. It must be designed to transfer the incident solar radiation to the receiver searching the maximal optical efficiency achievable with in an angular range limited by physical constrains[11]. The concentrating optics should produce a concentrated flux with reduced non-uniformity on the receiver, in order to minimize ohmic losses[12,13], and should be designed with great attention to many aspects related to its final industrial application. These are, in particular: compactness, tolerance on assembling errors, low cost of manufacturing processes, optimal placement of the receiver for electrical and thermal issues, use of materials which can guarantee high reliability, high durability and low cost, high efficiency at the module and

* Corresponding author:

parretta@fe.infn.it (Antonio Parretta)

Published online at http://journal.sapub.org/optics

Copyright (C 2012 Scientific \& Academic Publishing. All Rights Reserved array level.

This last point is related to the efficiency parameter, useful for a direct comparison with the standard flat-plate photovoltaics, calculated on the total intercepting area of the array, and not just, as sometimes it is found for CPV products, based on the efficiency of the optically active area. Indeed, this way to indicate the efficiency on the optical active area, useful for the design of the optics, may have a poorer impact on the system installation, where the most interesting aspects are the peak power achievable from each tracker of optimized cost, the land requested for total installed peak power and the achievable $\mathrm{kWh} / \mathrm{kWp}$. Pay attention to these aspects could have a fundamental impact on the final project of a CPV system; indeed, arrays with a relevant inactive/active optical area ratio risk to reduce the advantages of the CPV technology. All the previously indicated characteristics must be considered in the design of photovoltaic concentrators; many optical configurations have been proposed during the last years[1-5]; a large spectrum of possible designs, with different levels of effectiveness, can be achieved by applying the "nonimaging" optics[14-16].

The fundamental quantities of a PV solar concentrating optics usually considered are the geometric concentration ratio and the optical efficiency, giving the optical concentration ratio, and the flux distribution on the receiver. These quantities are defined on the basis of the irradiation conditions, that is on the angle-resolved radiance of the light source. At mid-high concentration ratios, that means at collecting angles of the order of few degree or less, only the direct component of solar radiation is effective, and the 
input flux can be approximated by a parallel beam of known spectral irradiance and direction. The optical efficiency and flux distribution on the receiver should be evaluated therefore as function of the orientation of the beam respect to a reference frame joint with the concentrator, and as function of wavelength, if the experimental apparatus is provided for spectral measurements. We have in this way introduced the concept of "optical characterization" of a solar concentrator[17-21]. Different approaches can be followed to perform it; here we recall the most important methods, directly derived by our recent research on this subject[22-30]: the "direct" method and the "inverse" method, depending on the way the concentrator is irradiated, if from the input or the output aperture, respectively. In the "direct" method, the angle-resolved transmission efficiency is obtained irradiating the input aperture by a suitably oriented parallel beam, of known irradiance, and measuring the output flux; this has to be repeated for all the significant directions of incidence, which are strictly dependent on the symmetry of the concentrator. From the transmission efficiency curves obtained for the different azimuthal directions an "acceptance angle" is derived as a parameter that defines the angular limit within which the incident radiation is collected with a minimum value of efficiency. An alternative way to obtain the angle-resolved optical efficiency is the "inverse" method, a very effective method where the concentrator can be tested irradiating it from the output aperture, therefore reversing the light path which occurs during the normal operating conditions. It is characterized by a remarkable rapidity of measurements and by a very simple apparatus with respect to the direct method[22-30]. The main features of this method are summarized in the next section and compared with another "inverse" method, derived from a modification of the original one[31,32]. The two "inverse" methods are applied to a recently developed nonimaging photovoltaic concentrator, used in CPV modules and named Rondine ${ }^{\circledR}$. The main purpose of this work is to show that, when the two inverse methods are suitably configured, they are able to give, by optical simulation, the same results in terms of the angle-resolved optical efficiency of the Rondine concentrator. Purpose of this work is also to show the advantages of the original inverse method in terms of simplicity and rapidity of simulation measurements. The same advantages of the original inverse method are expected for experimental measurements.

\section{The "Inverse" Methods of Optical Characterization}

The original inverse method developed by A. Parretta et al.[22-30] for the characterization of solar concentrators is the most simple among the many methods based on measurements with a CCD camera. It involves the use of a lambertian and unpolarized source of light (ls) to be positioned at the output aperture of the solar concentrator (sc), in place of the receiver, for irradiating it in a reverse way, a planar lambertian screen (ps) to intercept the light at a far distance $d$, and a CCD camera for recording the image produced on the screen (see Fig. 1a). The inverse method was originally developed for PV solar concentrators, in which the receiver, a solar cell, is a planar device. For receivers with non planar geometry, the lambertian source has to be shaped to reproduce the profile of the receiver surface. When the inverse method is simulated, the planar screen is configured as an ideal absorber and the measured incident irradiance $E(\theta, \varphi)$ (see Fig. 1b) is converted into the radiance distribution function of the concentrator, $L_{i n v}(\theta, \varphi)$, by the $(\cos \theta)^{-4}$ factor. Indeed, if $\mathrm{P}(\theta, \varphi)$ is a point on the screen, $E(\theta, \varphi)$ the corresponding incident irradiance and $d S$ an elementary area around $\mathrm{P}(\theta, \varphi)$, the flux through area $d S$ is $d \Phi=E(\theta, \varphi) \cdot d S$ and it is confined within the solid angle $d \Omega$ given by:

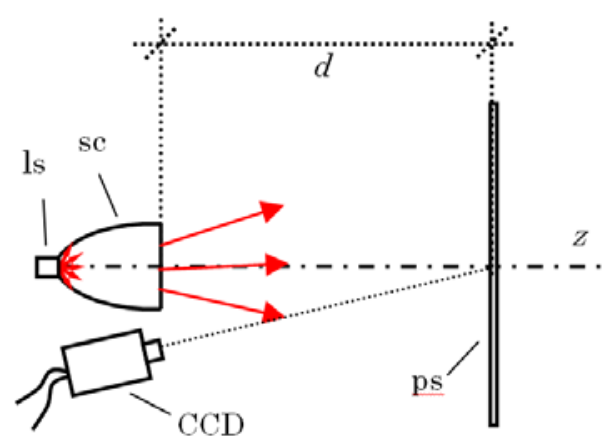

a)

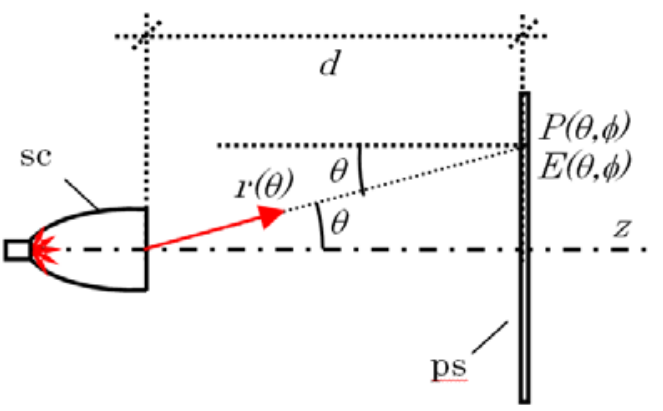

b)

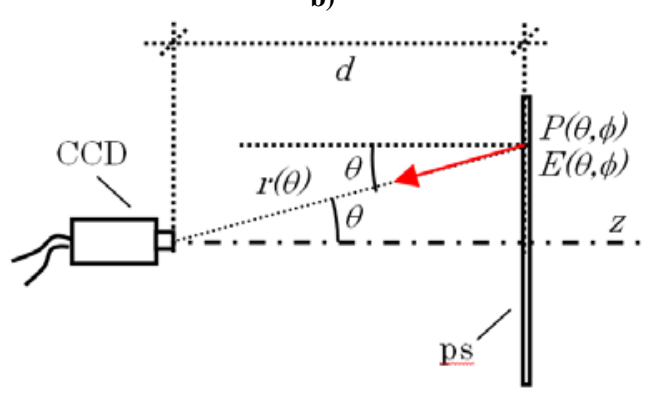

c)

Figure 1. (a) Schematic of the original "inverse" method; (ls): lambertian source; (sc): solar concentrator; (ps): planar screen. The CCD is shown inclined respect to the optical axis, but in reality it is almost aligned with it and close to the concentrator. (b) Schematic of the irradiation of the screen (ps) by the inverse light produced by the concentrator (sc). (c) Process of recording by the CCD of the image produced by the irradiance map on the planar screen (ps). $\mathrm{P}(\theta, \phi)$ is a point on the screen and $E(\theta, \phi)$ is the corresponding incident irradiance 


$$
d \Omega=\frac{d S \cdot \cos \theta}{r^{2}(\theta)}=\frac{d S \cdot \cos \theta}{(d / \cos \theta)^{2}}=\frac{d S}{d^{2}} \cdot \cos ^{3} \theta
$$

The inverse radiance produced by the concentrator towards $(\theta, \varphi)$ direction will be therefore expressed by:

$$
\begin{aligned}
& L_{i n v}(\theta, \phi)=\frac{d \Phi}{d \Omega \cdot A_{\text {in }} \cdot \cos \theta} \\
& =\frac{E(\theta, \phi) \cdot d S}{\left(d S \cdot \cos \theta / r^{2}\right) \cdot A_{\text {in }} \cdot \cos \theta}=\ldots \\
& \ldots=\frac{E(\theta, \phi) \cdot d S}{\left(d S \cdot \cos ^{3} \theta / d^{2}\right) \cdot A_{\text {in }} \cdot \cos \theta}=\frac{d^{2}}{A_{\text {in }}} \cdot \frac{E(\theta, \phi)}{\cos ^{4} \theta}
\end{aligned}
$$

where $A_{i n}$ is the input aperture area of concentrator. The radiance can be normalized to the value at $\theta=0^{\circ}$ giving:

$$
L_{\text {inv,norm }}(\theta, \phi)=\frac{L_{\text {inv }}(\theta, \phi)}{L_{\text {inv }}(0)}=\frac{E(\theta, \phi)}{E(0) \cdot \cos ^{4} \theta}=\frac{E_{\text {norm }}(\theta, \phi)}{\cos ^{4} \theta}(3)
$$

If no scattering or interference/diffraction phenomena occurs, the reversibility principle of the light paths can be applied[33], which establishes the invariance of attenuation undergone by an unpolarized e.m. radiation at reflective or refractive interfaces, reversing the optical path direction. Starting from this principle, it can be demonstrated[22,27,28] that angular distribution of the inverse radiance $L_{\text {inv }}(\theta, \varphi)$ reproduces the angular distribution of the optical transmission efficiency $\eta_{\text {dir }}(\theta, \varphi)$ in "direct" irradiation mode; thus the two normalized functions coincide:

$$
\begin{aligned}
& L_{i n v, \text { norm }}(\theta, \phi)=\frac{L_{i n v}(\theta, \phi)}{L_{i n v}(0)} \\
& =\eta_{\text {dir }, \text { norm }}(\theta, \phi)=\frac{\eta_{\text {dir }}(\theta, \phi)}{\eta_{\text {dir }}(0)}
\end{aligned}
$$

We can conclude that the normalized pro file of the direct transmission efficiency is directly derived by the normalized irradiance incident on the screen, by the expression:

$$
\eta_{\text {dir }, \text { norm }}(\theta, \phi)=\frac{E_{\text {norm }}(\theta, \phi)}{\cos ^{4} \theta}
$$

When the "inverse" method is applied experimentally, the screen is used to send back the diffuse, inverse light towards the CCD and must have a Lambertian character (reflectivity independent on the incidence angle at constant incident flux, and constant radiance of the reflected light, as function of observation angle) in order to allow the reconstruction of the irradiance map on the screen from the intensity map on the CCD. If the CCD is aligned with the optical $z$ axis and close to the concentrator, the intensity profile of CCD image must be corrected by a further $(\cos \theta)^{-4}$ factor. This is illustrated in Fig. 1c where the CCD is perfectly aligned with the optical axis of the concentrator. The intensity $I(\theta, \varphi)$ on the CCD and its corresponding normalized function $I_{\text {norm }}(\theta, \varphi)$ can be expressed as function of the incident irradiance $E(\theta, \varphi)$ on the screen as follows:

$$
\begin{gathered}
I(\theta, \phi)=\text { const } \cdot \frac{E(\theta, \phi) \cdot R \cdot \cos ^{2} \theta}{r^{2}(\theta)} \\
=\text { const } \cdot \frac{E(\theta, \phi) \cdot R \cdot \cos ^{4} \theta}{d^{2}} \\
I_{\text {norm }}(\theta, \phi)=\frac{I(\theta, \phi)}{I(0)} \\
=\frac{E(\theta, \phi) \cdot \cos ^{4} \theta}{E(0)} \\
=E_{\text {norm }}(\theta, \phi) \cdot \cos ^{4} \theta
\end{gathered}
$$

where $R$ is the screen reflectivity (independent of $\theta, \varphi$ because the screen is lambertian, and dependent only on the wavelength). From Eqs. (3), (4) and (6.2) we finally obtain the previously anticipated result for the transmission efficiency:

$$
\eta_{\text {dir, norm }}(\theta, \phi)=I_{\text {norm }}(\theta, \phi) \cdot \cos ^{-8} \theta
$$

The "inverse" method is very fast and easy to apply, if compared to the conventional "direct" method; it shows some limitations in experimental indoor measurements on large concentrators, because the distance $d$ must be kept well higher than the linear size of input aperture $\left(d>>\sqrt{ } A_{i n}\right.$; $A_{\text {in }}$ input aperture area) in order to have a good angular resolution $\Delta \theta$ from the CCD image, being $\Delta \theta$ expressed as:

$$
\Delta \theta \approx \operatorname{tg}^{-1}\left[\sqrt{A_{\text {in }}} \cdot \cos ^{2} \theta / 2 d\right]
$$

The optical characterization of a solar concentrator implies the drawing of its transmission efficiency curve $\eta_{\text {dir }}(\theta, \varphi)$. The angular resolution must be smaller than the acceptance angle, $\Delta \theta<<\theta_{a c c}$, with $\theta_{a c c}$ defined as the angle at which the trans mission efficiency drops to the $50 \%$ of the value at $0^{\circ}[15,16]$. For photovoltaic applications, the acceptance angle is conventionally defined in a different way, i.e. the angle at which the transmission efficiency drops to the $90 \%$ of the value at $0^{\circ}$. The "inverse method" allows to get also the absolute transmission efficiency function $\eta_{d i r}(\theta, \phi)$ after performing the measure of $\eta_{\text {dir }}(0)$ (see Eq. (4))[22], resulting in this way completely alternative to the "direct method". To this purpose, a modification of the measuring configuration is required: the lambertian screen is removed and replaced by the CCD oriented towards the input aperture of concentrator, as illustrated in Fig. 2a. The CCD records the image of the input aperture, which is elaborated in order to have the average intensity of the total aperture area and the average intensity of only the lambertian source area (see Fig. 2b). These two quantities are respectively proportional to the average radiance of the total aperture, $L_{\text {inv }}(0)$, and to the average radiance of the lambertian source, $L_{i n v}$. The on-axis transmission efficiency, $\eta_{\text {dir }}(0)$, is given by the ratio between the two radiances, following a theoretical treatment presented in ref.[22]:

$$
\eta_{\text {dir }}(0)=\frac{L_{i n v}(0)}{L_{i n v}}
$$


Fig. 2b shows, as an example, the image of input aperture of the Rondine concentrator, in its first, Gen 1, version (see next section) irradiated in the inverse way. The central rectangular area corresponds to the lambertian source, more intense respect to the rest of aperture area, as it is expected to have $\eta_{\text {dir }}(0)<1$. The grey zones located around the output openings show that the irradiance emitted from there on the optical axis direction is lower compared to the rest of surface; this fact, for the basic principle of "inverse" method, means that the same area, if irradiated in "direct" mode from a beam parallel to the optical axis, should reflect less light toward the exit opening, that is it has a lower optical transmission efficiency, respect to the rest of surface. This area of reduced efficiency is consequence of the surface deformation generated in the molding fabrication process.

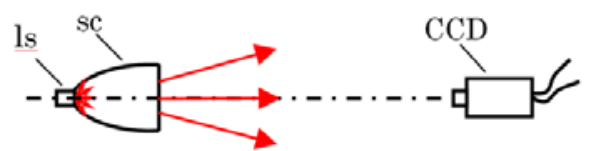

a)

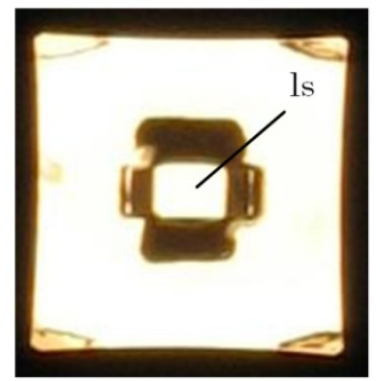

b)

Figure 2. a) Schematic of the "inverse" method for measure of $\eta_{\text {dir }}(0)$; b) real image of the input aperture of the Rondine Gen1 as seen by the CCD

Purpose of this work is to compare the just discussed original inverse method (here referred to as Parretta method or P-method) with a method derived by the "luminescence" method, recently introduced by R. Herrero et al.[31,32]. To develop the "luminescence" method, Herre ro et al. modified the P-method with the aim to test the real optical properties of a photovoltaic solar concentrator as a whole: optical unit + receiver (the solar cell). In this approach the electroluminescence (EL) light emitted by the forward biased solar cell (the receiver of concentrator) acts as reverse light. Here, the "generalized" Kirchhoff's law must be applied, which was derived by Wurfel[34] by applying a thermodynamic treatment to both thermal and non-thermal radiation and which is based on the concept of the chemical potential of the radiation. From the generalized Kirchhoff's law, the solar cell contributes to the reverse light with the same efficiency with which it contributes to the absorption of light under direct irradiation[35]. The second change made by Herrero et al. to the P-method was the use of a parabolic mirror to focus the EL light on the lambertian screen[31,32]. This choice allows to get the polar distribution of the transmission efficiency directly on the screen, avoiding the need to keep the screen far from the concentrator.

The peculiarity of the "luminescence" method is that it operates with "real" receivers, not the "ideal" lambertian ones (with unitary absorptivity); while this is a good feature for the characterization of specific CPV optics, it limits the method to photovoltaic solar concentrators. To have general methods for testing any type of solar concentrator, independently from the nature of receiver (photovoltaic or thermodynamic), a source of lambertian properties for the reverse light is required. The use of a parabolic mirror to focus the reverse light on the screen is generally useful, so in this work we explo it the idea of adding a parabolic mirror to the optical path of reverse light in the original inverse method. The result is not really a new method; however we refer to it as the $\mathrm{PH}$ (Parretta-Herrero) method, to keep memory of the two contributions. The schematic of PH-method is reported in Fig. 3. Here, the raytracing of rays emerging parallel to the axis of the concentrator and focused on the origin of the $\mathrm{x} / \mathrm{y}$ frame fixed on the screen is shown. The solar concentrator (sc) is not visible because it is much smaller of the parabolic mirror $(\mathrm{pm})$ and of the planar screen (ps). In Fig. 3 the polar diagram built on the screen (ps) is also shown. The point of coordinates $(\theta, \varphi)$ is the target of any ray exiting from the concentrator at the same polar and azimuthal angles, independently from the starting position from the input aperture.

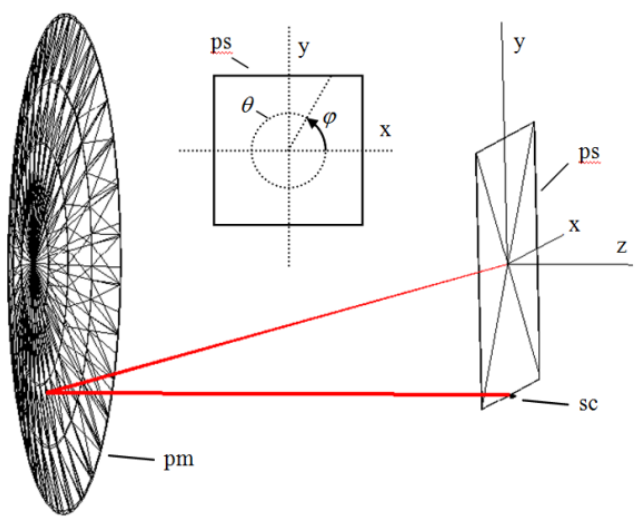

Figure 3. Schematic principle of the Parretta-Herrero $(\mathrm{PH})$ method used for simulating the optical properties of a generic solar concentrator. Rays exiting from different points of (sc) at the same polar angle $\theta$ and azimuthal angle $\varphi$, converge on the same point on the screen[31,32]

\section{Design Parameters and Calibration}

The optics of the photovoltaic concentrators used in this test of the characterization methods has been developed by CPower[36] and is called Rondine concentrator[37-42]. This optics has been selected because of its specific features, which permit to put in evidence all the performances of the inverse methods without reducing the generality of the characterization approach. Indeed, this optics is of small scale (few centimeters of lateral inlet aperture), it intercepts all the light emitted from the lambertian source and has smooth surfaces. These concentrators were developed by applying techniques of the nonimaging optics[14-16]; two 
types of concentrators, with different shape, concentration and dimensions were developed and manufactured: Rondine Gen1 and Rondine Gen2 (see Fig. 4); both of them have medium-low concentration levels, of $25 \mathrm{x}$ and $20 \mathrm{x}$ respectively. The basic idea for their design comes from the curvatures of CPC, with surfaces deformed in order to satisfy the electrical and mechanical constrains related to other characteristics required by the CPV module. Some aspects are similar to the CPC, because of the ideal optical peculiarities of this reference optics. The frontal aperture of these concentrators is squared, to allow a $100 \%$ filling factor in a rectangular module. The final effect is a concentrating unit with a peculiar four-pointed shape obtained by the removal of the four lateral walls produced by the squaring process. On each unit, the presence of four lateral walls parallel to the optical axis is not useful when the unit is embedded in the module; indeed, it should have the disadvantage of leading further losses of light energy for reflection on the non ideal surfaces.

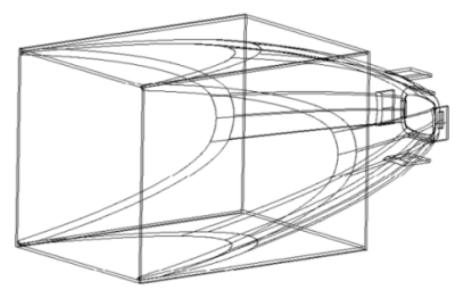

a)

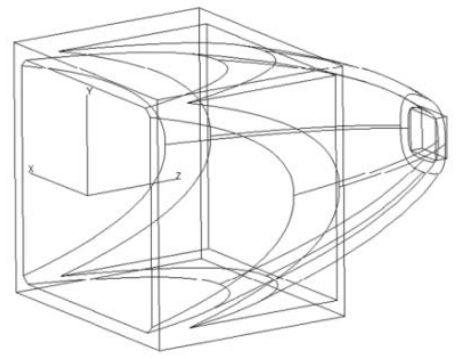

b)

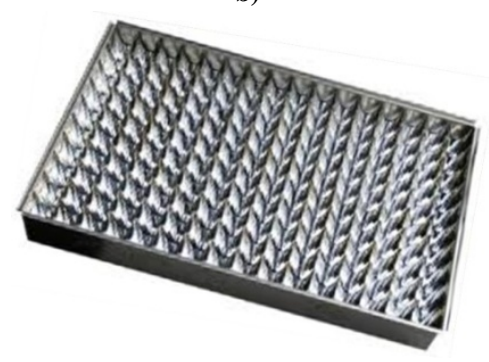

c)

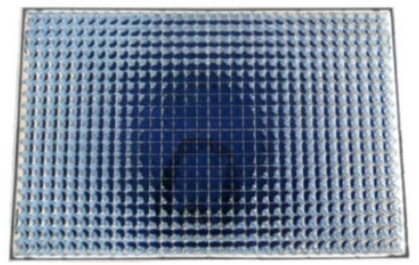

d)

Figure 4. a) CAD view of the boxed Rondine ${ }^{\circledR}$ Gen1. b) CAD view of the boxed Rondine ${ }^{\circledR}$ Gen2. c) Photo of the Rondine Gen 1 CPV module (Wp: $=95 \mathrm{~W}$; Eff.: $=12 \% \mathrm{C}=20 \mathrm{X}$ ). d) Photo of the Rondine Gen $2 \mathrm{CPV}$ module $(\mathrm{Wp}:=120 \mathrm{~W}$; Eff.: $=16 \% \mathrm{C}=20 \mathrm{X})$
The Rondine design permits to have some significant advantages: smooth surfaces facilitating the molding processes and improving the production yield; high angular acceptance; an optics directly connected to the receiver, which permits to avoid energy losses due to possible deformations of the frontal covering surface of the module; reflective optics produced with well established technology for cars' headlamps; possibility to achieve optical efficiency $>95 \%$ with Ag metallization. The Rondine Gen2 is shorter than Rondine Gen1, but not just scaled; it has a different curvature and output aperture almost squared (in both cases the exit apertures have round corners, as shown in Fig. 5). Their optical properties are similar, with an average surface reflectance of $\approx 0.85$ and an average acceptance angle of $\approx 4^{\circ}$. These optics are used in CPV modules mounted on two-axis solar trackers with pointing accuracy generally achieved for applications with standard, flat-plate PV panels (see Figs. 4c, d). Indeed, the pointing precision declared by many tracker producers for flat plate photovoltaic modules is usually below $\pm 2^{\circ}$. At this limit error, the relative optical efficiency of the Rondine optics is still of about $100 \%$, as it will be shown below. Accurate tracking systems usually employed for high concentration technologies permit to track the Sun with an accuracy < $\pm 0.1^{\circ}$. This high precision is not requested for the modules with the optics here considered. Moreover, the high angular acceptance permits higher tolerance for the module assembling in the arrays and less rigidity of the mechanical structures, with advantages for the reliability and for the availability of the installation. The CADs of Rondine Gen1 and Gen2 were imported in TracePro ${ }^{\circledR}$, the opto-mechanical design software of Lambda Research, and boxed with four ideal mirror walls (see Figs. 4a, b).

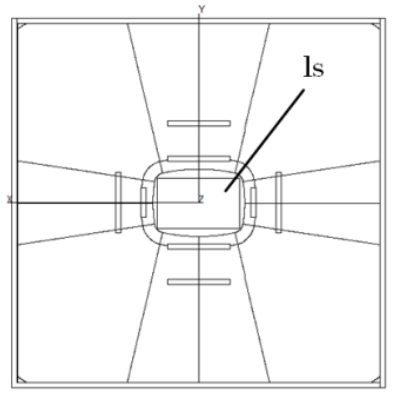

a)

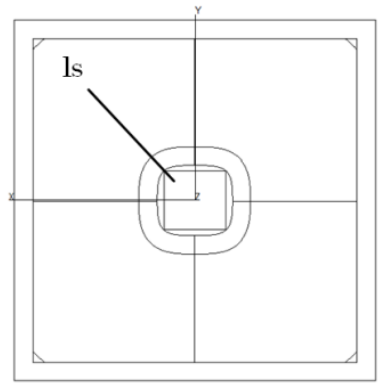

b)

Figure 5. Front view of the Rondine Gen1 (a) and Rondine Gen2 (b) input aperture, showing, at the center, the exit opening with inscribed the rectangular Lambertian source (ls) 


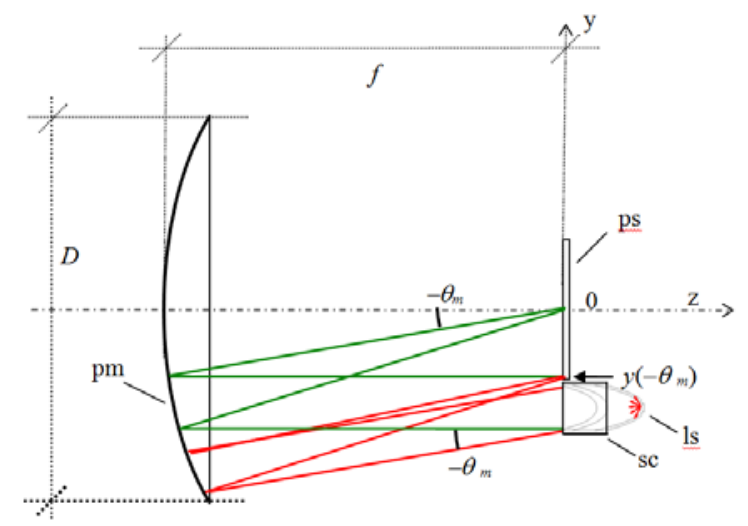

a)

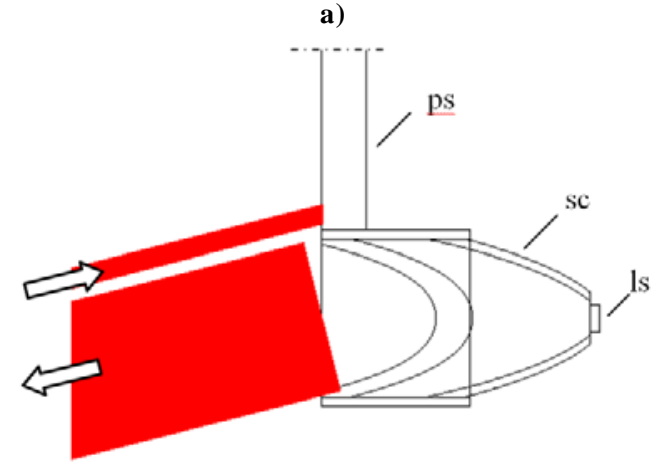

b)

Figure 6. a) Schematic principle of the Parretta-Herrero $(\mathrm{PH})$ method (Pm): parabolic mirror, (ps): planar screen, (sc): solar concentrator, (ls): lambertian source. $D$ and $f$ : diameter and focal length of (pm), respectively; l: side of the screen. The concentrator lies below the coordinate $y\left(-\theta_{\mathrm{m}}\right)$ in such a way to avoid any interference with the rays exiting at $\theta>-\theta \mathrm{m}$. b) It is shown a parallel beam emitted at $\theta=-\theta \mathrm{m}$ on the $y / z$ plane, which focuses at the bottom edge of the screen (ps)

The two concentrators were coupled to the lambertian sources (ls), a rectangle of $15.8 \times 10-\mathrm{mm}^{2}$ area for the Rondine Gen 1 and of $6.7 \times 6.5-\mathrm{mm}^{2}$ area for the Rondine Gen2; both rectangles were inscribed inside of the exit aperture of the concentrators (see Fig. 5). The optical efficiency curves derived by the inverse methods will be referred therefore to these inscribed areas, instead of to the total area of exit apertures. The area of the lambertian source corresponds therefore to that of a solar cell inscribed in the exit aperture, as used in the direct characterization method on these optics.

The two concentrators were inversely ray-traced following the P-method (see Fig. 1) and the PH-method (see Figs. 3 and 6), by using 500k rays, each ray of $1-\mathrm{W}$ flux. In the P-method the reverse light of Rondine Gen1 was projected onto a square screen (ps) of 16,000 -mm side placed at 30,000-mm distance from input aperture; in this way the inverse light was collected with in $\pm 15^{\circ}$ along the two orthogonal directions ( $x$ and $y$ ), parallel to the edges of the input aperture. The angular resolution is that obtained by applying Eq. (8), where $\sqrt{ } A_{i n}=70 \mathrm{~mm}$ for Rondine Gen1 and $\sqrt{ } A_{\text {in }}=35 \mathrm{~mm}$ for Rondine Gen2. The resolution is better than $0.1^{\circ}$ for the Rondine Gen 1 and $0.05^{\circ}$ for the Rondine Gen2 for any direction of emitted radiation. The resolution is better than $0.07^{\circ}$ for the Rondine Gen 1 and $0.03^{\circ}$ for the Rondine Gen 2 along the $x / y$ directions. As it comes out from Eq. (8), the angular resolution is the worst at $\theta=0^{\circ}$ and improves at increasing $\theta$. The schematic configuration of PH-method is reported in Fig. 6a. The Rondine optics was deliberately enlarged to better show the path of the rays. The parabolic mirror (pm) has 6,120-mm aperture diameter $D$, 390-mm length, 6,000-mm focal length $f$, and the square absorber (ps) has 3,200-mm side $l$. The solar concentrator (sc) coupled to the la mbertian source (ls) is placed just below the screen, with its input aperture planar to the screen surface. The position of the concentrator was accurately calculated placing it at a suitable distance from the optical axis to avoid any interference with rays reflected by the mirror $(\mathrm{pm})$. This distance was found after fixing the maximum angular divergence, $\pm \theta_{m}$ (with $\theta_{m}>0$ ) of inverse rays to be recorded along the $\mathrm{x} / \mathrm{y}$ axes. For our simulations we have chosen $\theta_{m}$ $=15^{\circ}$, because at this angle the optical efficiency is sufficiently low ( $\approx 20 \%$ of the maximum value) and the optical efficiency curve sufficiently well defined. Fig. 6a simulates red rays emerging from the concentrator with divergence $-\theta_{m}$, which converge to the bottom of the screen $y\left(-\theta_{m}\right)$, just at the upper edge of the Rondine Gen2, after being reflected by mirror $(\mathrm{pm})$; this is highlighted in the enlarged detail of Fig. 6b. Fig. 6a shows also that all the rays (green) with direction parallel to the $z$ axis are focused at the center of the screen. The $y$ coordinate of rays emitted on the $y / z$ plane at angle $\theta_{m}$ can be expressed as:

$$
y\left(\theta_{m}\right)=\frac{2 f\left(1-\cos \theta_{m}\right)}{\sin \theta_{m}}
$$

Before simulating the Rondine concentrators with TracePro ${ }^{\circledR}$ software, the calibration of the angle coordinates in terms of $x$ and $y$ coordinates on the square screen (ps) was performed. This was done by setting a uniform and parallel source of light exiting from the input aperture of the Rondine concentrator (see Fig. 6b), oriented towards the mirror $(\mathrm{pm})$ at some calibrated polar angles measured on the $y / z$ incident plane, and on a plane parallel to the $x / z$ plane and crossing the centre of concentrator. Once a certain number of polar angles for the parallel beam were chosen, the $x / y$ coordinates of the point on the screen, where the beam was focused, were recorded. The calibration has provided the angle coordinate along $x$ and $y$ axes, as function of the pixel number $n_{p x}$ (maximum 128 for both axes covering the entire screen); for the Rondine Gen1 concentrator we obtain:

$$
\begin{gathered}
\theta_{X}\left({ }^{\circ}\right)=14.94-0.232 \cdot n_{p x} \\
\theta_{Y}\left({ }^{\circ}\right)=14.75-0.226 \cdot n_{p x}
\end{gathered}
$$

and for the Rondine Gen 2 concentrator we obtain:

$$
\begin{aligned}
& \theta_{X}\left({ }^{\circ}\right)=15.11-0.234 \cdot n_{p x} \\
& \theta_{Y}\left({ }^{\circ}\right)=14.75-0.224 \cdot n_{p x}
\end{aligned}
$$

The calibration has provided an excellent linear, and practically equal behavior for both axes. Fig. 7 shows, as an example, the calibration curve for the Rondine Gen 1. 


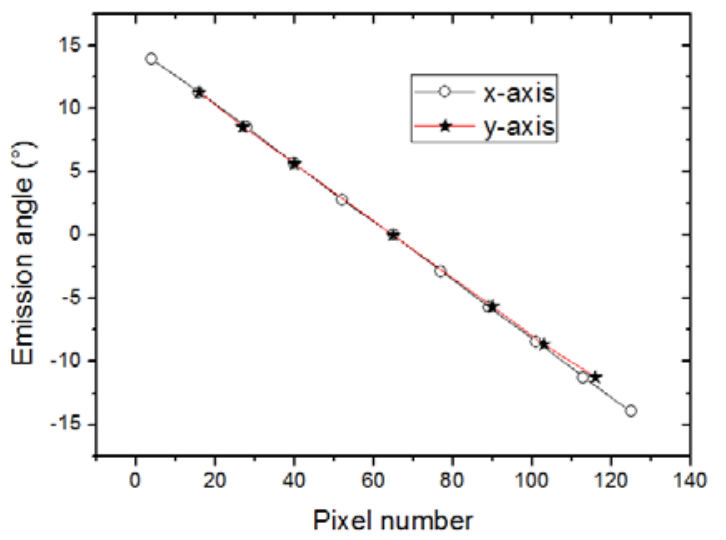

Figure 7. Calibration curves of the emission angle vs. pixel number relat ive to the $x$ and $y$ axes of the screen

\section{Optical simulations}

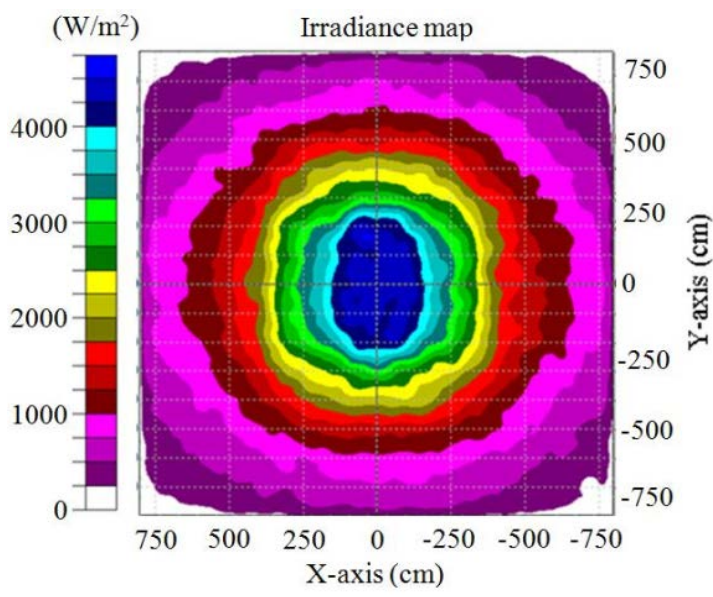

a)

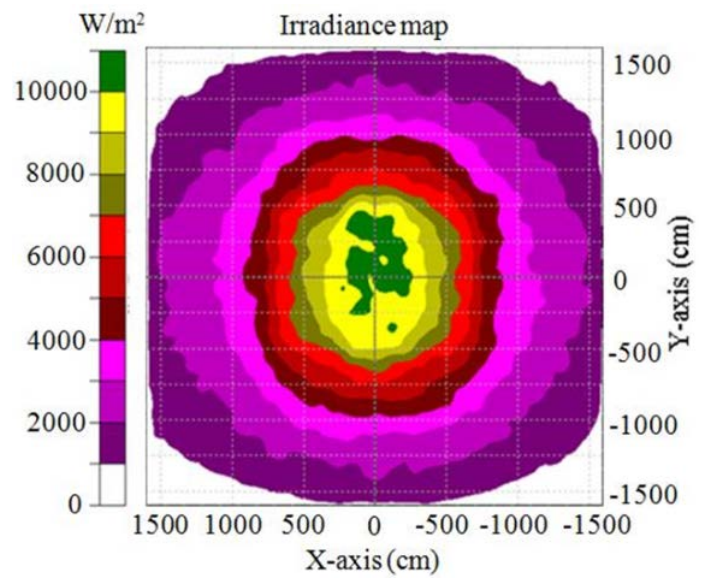

b)

Figure 8. $\quad$ Rondine ${ }^{\circledR}$ Gen1: a) flux distribution recorded on the screen (ps) for the P-method; b) flux distribution recorded on the screen (ps) for the PH-method

The flux distributions (irradiances in $\mathrm{W} / \mathrm{m}^{2}$ ) recorded for the P-method and the PH-method applied to Rondine ${ }^{\circledR}$ Gen1 are shown in Figs. 8a and 8b, respectively. The map of Fig. $8 b$ directly reproduces the radiance of the inversely irradiated concentrator, because of the reflection of the inverse light on the parabolic mirror surface. From the two flux distributions we derive the corresponding $x$-axis and $y$-axis profiles of the "normalized" transmission efficiency $\eta_{\text {dir,norm }}(\theta, \phi)$ as follows: for the P-method the "normalized" $x / y$-axes profiles of the intensity (irradiance) on the screen were multiplied by the $(\cos \theta)^{-4}$ factor; for the PH-method, on the contrary, they were directly obtained from the "normalized" $x / y$-axes profiles of the intensity (irradiance) on the screen. The $x / y$-axes profiles of the intensity on the screen were obtained by taking the average of the four central rows/columns of the intensity map, respectively, covering an angular interval of $0.5^{\circ}$. The $x$-axis and $y$-axis profiles of $\eta_{\text {dir,norm }}(\theta)$ derived by the two methods are shown in Figs. 9a and 9b, respectively. The efficiency data are reported with an uncertainty coming from the fluctuation of flux intensity in the $0.5^{\circ}$ angular interval. These fluctuations are due to the finite number of rays used for the simulations, so they could be further reduced by increasing the number of rays in the raytracing. The \pm values on the $x$-axis profile correspond to the $\varphi=0$ and $\varphi=\pi$ azimuth angles, respectively, whereas the \pm values on the $y$-axis profile correspond to the $\varphi=\pi / 2$ and $\varphi$ $=3 \pi / 2$ azimuth angles, respectively. Fig. 9 shows that the $x$-axis profile of the Rondine Gen 1 is more pointed than the $y$-axis profile, as an effect of the longer $x$-axis side of the exit aperture (see Fig. 5a).

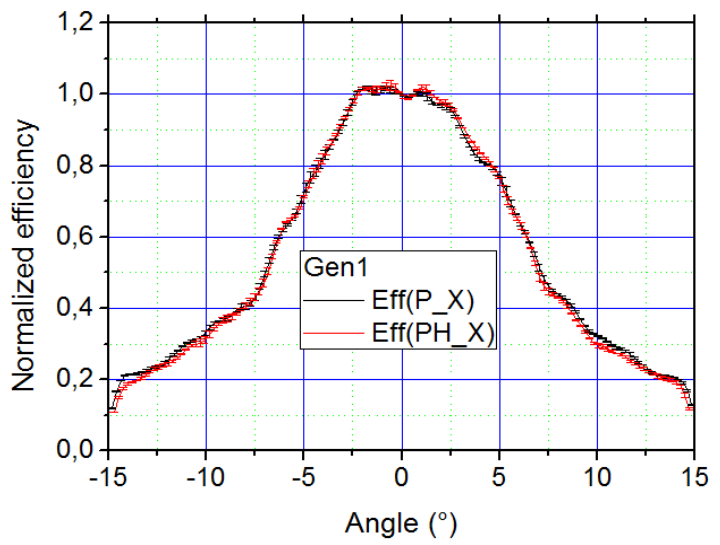

a)

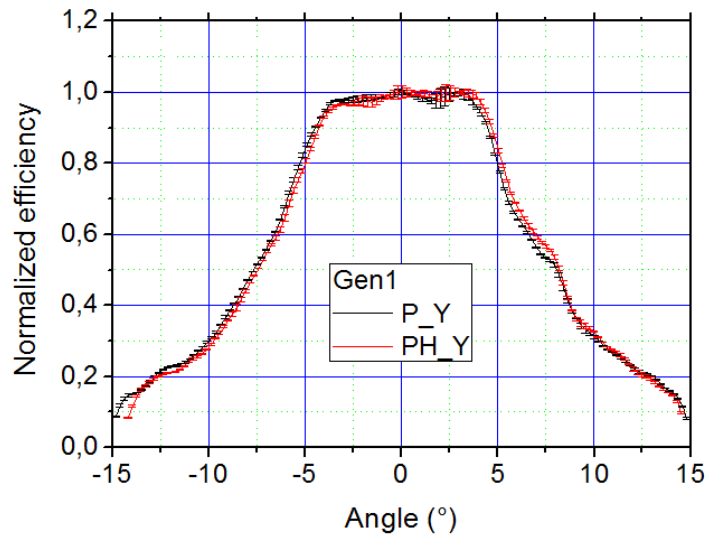

b)

Figure 9. Rondine $^{\circledR}$ Gen1: $X$-axis (a) and $y$-axis (b) of the normalized optical efficiency profiles derived by the P-method and the PH-method 
The flux distributions recorded for the P-method and the PH-method applied to Rondine ${ }^{\circledR}$ Gen 2 are shown in Figs. $10 \mathrm{a}$ and $\mathrm{b}$, respectively. The corresponding $x$-axis and $y$-axis profiles of the normalized transmission efficiency $\eta_{\text {dir,norm }}(\theta)$ are shown in Figs. $11 \mathrm{a}$ and b, respectively. Comparing the efficiency profiles of Figs. 9 and 11 referred to both concentrators, the excellent overlap of the profiles obtained with the two methods appears evident.

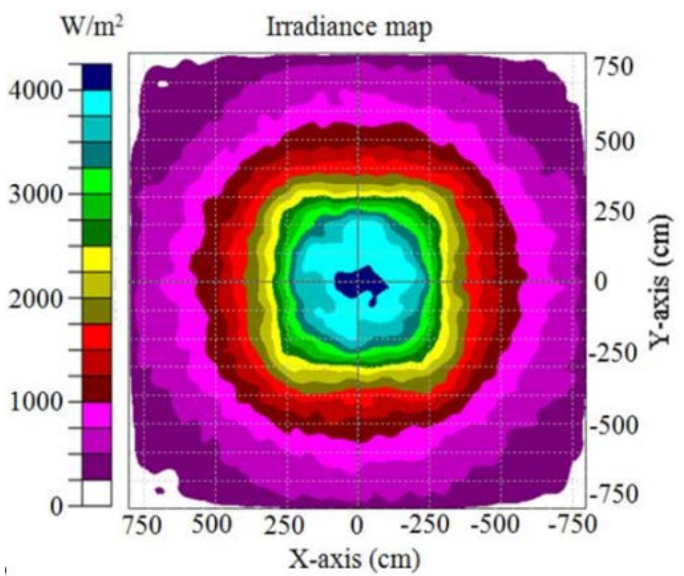

a)

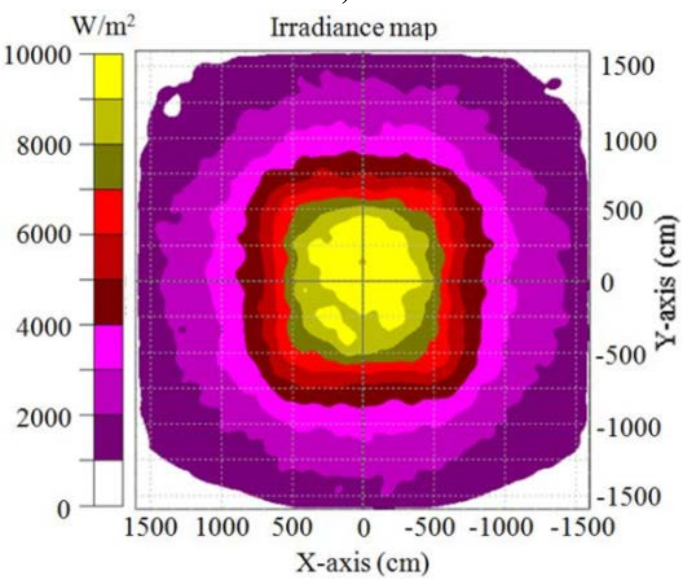

b)

Figure 10. Rondine ${ }^{\circledR}$ Gen2: a) flux distribution recorded on the screen (ps) for the P-method; b) flux distribution recorded on the screen (ps) for the PH-method

Table 1. Summary of average acceptance angles measured, at $50 \%$ and $90 \%$ of the maximum, on the $x$ and $y$ profiles of the transmission efficiency curves of the Rondine Gen 1 and Gen 2 concentrators by the P-method and PH-method

\begin{tabular}{|c|c|c|c|c|c|c|c|c|}
\hline \multirow{2}{*}{} & \multicolumn{3}{|c|}{ Rondine Gen1 } & \multicolumn{3}{c|}{ Rondine Gen2 } \\
\cline { 2 - 9 } & \multicolumn{2}{|c|}{ X-axis } & \multicolumn{2}{|c|}{ Y-axis } & \multicolumn{2}{c|}{ X-axis } & \multicolumn{2}{c|}{ Y-axis } \\
\cline { 2 - 9 } & $90 \%$ & $50 \%$ & $90 \%$ & $50 \%$ & $90 \%$ & $50 \%$ & $90 \%$ & $50 \%$ \\
\hline \multirow{2}{*}{ P-method } & 3.10 & 6.99 & 4.52 & 7.88 & 4.08 & 6.71 & 4.16 & 6.74 \\
\hline \multirow{2}{*}{ PH-method } & 3.10 & 6.91 & 4.53 & 7.83 & 4.08 & 6.60 & 4.17 & 6.51 \\
\hline
\end{tabular}

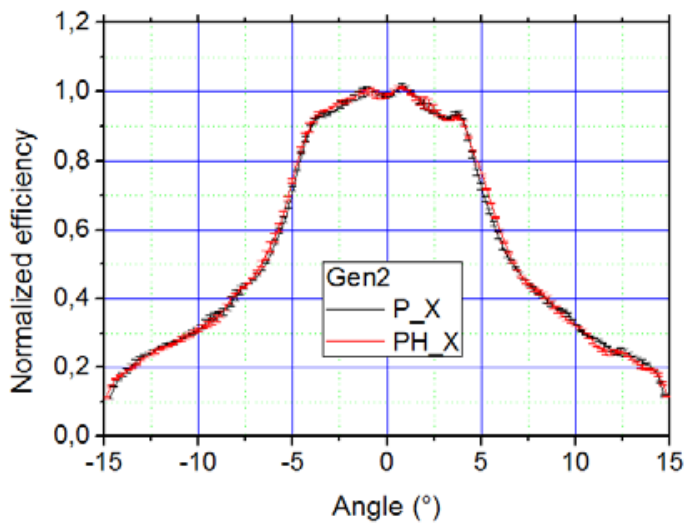

a)

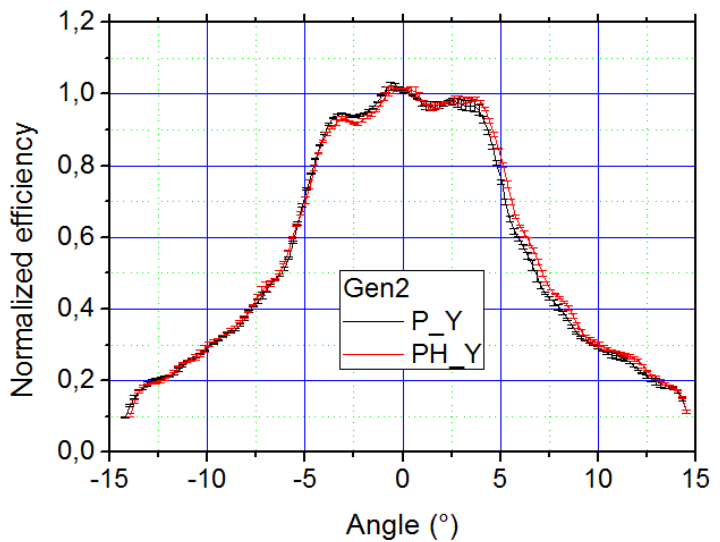

b)

Figure 11. Rondine ${ }^{\circledR}$ Gen2: $X$-axis (a) and $y$-axis (b) of the normalized optical efficiency profiles derived by the P-method and the PH-method

The coma aberration effects expected for the $\mathrm{PH}$-method due to the presence of the parabolic mirror is therefore negligible, as previously verified during the calibration of angles. Table 1 summarizes the average acceptance angle values obtained at $50 \%$ and $90 \%$ of the $0^{\circ}$ efficiency, reported for all the discussed simu lations. Tab. 1 also shows that the acceptance angles obtained for the two methods coincide within $0.1^{\circ}$. 
After the experience learnt with this work, we can affirm that the PH-method, when used for simulations, is rather more complex to apply respect to the classic inverse method (P-method). Critical points of the PH-method are: i) the position of concentrator must be calculated with high accuracy for the most divergent rays; ii) the parabolic mirror must be dimensioned in order to not reflect rays inside the concentrator; iii) the angle calibration on the flux map is required and is a quite long procedure. The above three steps require a not negligible optimization procedure. The P-method is not subject to this type of limitations, mainly because the (sc) is aligned with the $\mathrm{z}$ axis and the radiance of concentrator, whose profile is the same of the optical efficiency, is easily extracted from the irradiance distribution on the planar screen.

\section{Conclusions}

We have demonstrated the equivalence between two inverse methods of optical characterization of solar concentrators, the P-method and the PH-method. They were applied to two specific optics for PV solar concentrators, the Rondine ${ }^{\circledR}$ Gen 1 and Gen2 nonimaging concentrators. The two methods were not specifically applied to particular solar cell devices, requiring evaluation of luminescence effects. The first inverse method (P-method) remains the most simple, fast and reliable for simulating any type of solar concentrator (photovoltaic or thermodynamic) and for indoor experimental measurements on small-size concentrators. The PH-method is more suitable for laboratory experimental measurements of large concentrators, requiring a reduced size of the characterization system, but introducing a complex calibration procedure and expensive equipment.

\section{REFERENCES}

[1] A. Luque, S. Hegedus, "Handbook of Photovoltaic Science and Engineering”, John Wiley \& Sons Ltd, Chichester, UK, 2011 (ISBN: 978-0-470-72169-8).

[2] A. Luque, V.M. Andreev, "Concentrator Photovoltaics", Springer-Verlag, Berlin, D, 2007 (ISBN: 978-3-540-68796-2 ) .

[3] A. Martí, A. Luque, "Next Generation Photovoltaics”, Institute of Physics, Bristol, UK, 2004 (ISBN: 0-7503-0905-9).

[4] V.M. Andreev, V.A. Grilikhes, V.D. Rumyantsev, "Photovoltaic Conversion of Concentrated Sunlight", John Wiley \& Sons, Chichester, UK, 1997 (ISBN: 0-471-96765-3).

[5] A. Luque, "Solar Cells and Optics for Photovoltaic Concentrators”, Institute of Physics, Bristol, UK, 1989 (ISBN: 0-85274-106-5).

[6] D. Barlev, R. Vidu, P. Stroeve, “Innovations in concentrated solar power", Solar Energy Materials \& Solar Cells, 95, pp. 2703-2725, 2011.

[7] T.M. Razikov, C.S. Ferekides, D. Morel, E. Stefanakos, H.S. Hullal, H.M. Upadhyaya, "Solar photovoltaic electricity: Current status and future prospects”, Solar Energy, 85, 1580-1608, 2011.

[8] J.S. Coventry, “A solar concentrating photovoltaic/thermal collector”, Ph.D.Thesis, Australian National University, Canberra, AUS, 2004.

[9] D. Mills, "Advances in solar thermal electricity technology", Solar Energy, 76, pp. 19-31, 2004.

[10] S.A. Kalogirou, "Solar thermal collectors and applications" Progresses in Energy and Combustion Science, 30, pp. 231-295, 2004.

[11] G. Smestad, H. Ries, R. Winston, E. Yablonovich, “The thermodynamic limits of light concentrators, Solar Energy Materials, 21, pp. 99-111, 1990.

[12] A. Antonini, M. Stefancich, D. Vincenzi, C. Malagù, F. Bizzi, A. Ronzoni, G. Martinelli, "Contact grid optimization methodology for front contact concentration solar cells", Solar Energy Materials and Solar Cells, 80, pp. 155-166, 2003.

[13] D. Vincenzi, M. Stefancich, S. Baricordi, M. Gualdi, G. Martinelli, A. Parretta, A. Antonini, "Effects of irradiance distribution uneveness on the ohmic losses of CPV receivers”, Proc. of 24 ${ }^{\text {th }}$ EU PVSEC, pp. 725-728, 2009.

[14] J. Chaves, “Introduction to Nonimaging Optics”, CRC Press Taylor \& Francis Group, Boca Raton, USA, 2008 (ISBN: 978-1-4200-5429-3).

[15] R. Winston, J.C. Miñano, P. Benítez, “Nonimaging Optics”, Elsevier Academic Press, Amsterdam, NL, 2005 (ISBN: 978-0-12-759751-5).

[16] W.T. Welford, R. Winston, "High Collection Nonimaging Optics”, Academic Press Inc, San Diego, USA, 1989 (ISBN: 0-12-742885-2).

[17] P. Sansoni, F. Francini, D. Fontani, “Optical characterization of solar concentrator”, Optics and Lasers in Engineering, 45, pp. 351-359, 2007.

[18] A. Parretta, F. Aldegheri, D. Roncati, C. Cancro, R. Fucci, "Optical Efficiency of "PhoCUS" C-M odule Concentrators", Proc. 26 ${ }^{\text {th }}$ EU PVSEC, European Commission JRC, pp. 658-663, 2011

[19] A. Parretta, A. Antonini, M. Stefancich, V. Franceschini, G. Martinelli, M. Armani, "Characterization of CPC solar concentrators by a laser method”, Proc.of SPIE Optics and Photonics Conference, Ed. D.R. Myers, 6652, pp. 665207-1-12, 2007.

[20] J. Nilsson, “Optical design and characterization of solar concentrators for photovoltaics, Ph.D. Thesis, Lund University, Lund, S, 2005.

[21] M. Brogen, “Optical efficiency of low-concentrating solar energy systems with parabolic reflectors, Ph.D. Thesis, Acta Universitatis Upsaliensis, Uppsala, S, 2004.

[22] A. Parretta, D. Roncati, "Theory of the "inverse method" for characterization of solar concentrators”, Proc. of Optics for 
Solar Energy (SOLAR), Optical Society of America, STuE2, 2010.

[23] A. Parretta, G. Martinelli, A. Antonini, D. Vincenzi, "Direct and inverse methods of characterization of solar concentrators”, Proc. of Optics for Solar Energy (SOLAR), Optical Society of America, STuA1, 2010.

[24] A. Parretta, D. Roncati, L. Zampierolo, “Theoretical aspects of light collection in solar concentrators", Proc. of Optics for Solar Energy (SOLAR), Optical Society of America, STuE1, 2010.

[25] A. Parretta, A. Antonini, M. Butturi, E. Milan, P. Di Benedetto, D. Uderzo, P. Zurru, “Optical methods for indoor characterization of small-size concentrators prototypes", Advances in Science and Technology, 74, pp. 196-204, 2010.

[26] A. Parretta, G. Martinelli, E. Bonfiglioli, A. Antonini, M. Butturi, P. Di Benedetto, D. Uderzo, P. Zurru, E. Milan, D. Roncati, "Indoor optical characterization of the nonimaging “Rondine” PV solar concentrator", Proc of $24^{\text {th }}$ EU PVSEC, European Commission JRC, pp. 747-752, 2009.

[27] A. Parretta, A. Antonini, E. Milan, M. Stefancich, G. Martinelli, M. Armani, "Optical efficiency of solar concentrators by a reverse optical path method", Optics Letters, 33, pp. 2044-2046, 2008.

[28] A. Parretta, A. Antonini, M. Butturi, P. Di Benedetto, E. Milan, M. Stefancich, D. Uderzo, P. Zurru, D. Roncati, G. Martinelli, M. Armani, "How to "display" the angle-resolved transmission efficiency of a solar concentrator reversing the light path", Proc. of $23^{\text {rd }}$ EU PVSEC, European Commission JRC, pp. 95-98, 2008.

[29] A. Parretta, A. Antonini, M. Stefancich, G. Martinelli, M. Armani, "Inverse illumination method for characterization of CPC concentrators", Proc. of SPIE, Optics and Photonics Conference, Ed. D.R. Myers, 6652, pp. 665205-1-12, 2007.

[30] A. Parretta, A. Antonini, M. Stefancich, G. Martinelli, M. Armani, "Optical characterization of CPC concentrator by an inverse illumination method, Proc. of $22^{\text {th }}$ EU PVSEC, European Commission JRC, pp. 740-744, 2007.

[31] R. Herrero, C. Domínguez, S. Askins, I. Antón, G. Sala, "Two-dimensional angular transmission characterization of CPV modules”, Optics Express, 18 pp. A499-A505, 2010.
[32] R. Herrero, C. Domínguez, I. Antón, S. Askins, G. Sala, "Tools development for CPV characterization based on CCD camera measurements", Proc. of Optics for Solar Energy (SOLAR), Optical Society of America, STuA4, 2010.

[33] B. Rossi, “Optics”, Addison Wesley, Reading, UK, 1957.

[34] P. Würfel, “The chemical potential of radiation”, J. Phys. C: Solid State Phys., 15, pp. 3967-3985, 1982.

[35] L. Ferraioli, P. Maddalena, E. Massera, A. Parretta, M.A. Green, A. Wang, J. Zhao, Appl. Phys. Lett., 85, pp. 2484-2486, 2004.

[36] www.qower.it

[37] A. Antonini, M. Butturi, P. Di Benedetto, P. Zurru, D. Sartore, "In-Field performances of first commercial Rondine ${ }^{\circledR}$ PV concentrators”, Proc. of $26^{\text {th }}$ EU PVSEC, European Commission JRC, pp. 640-643, 2011.

[38] A. Antonini, M. Butturi, P. Di Benedetto, E. Milan, D. Uderzo, P. Zurru, D. Sartore, "GEN2 Rondine ${ }^{\circledR}$ PV Concentrators", Proc of $25^{\text {th }}$ EU PVSEC, European Commission JRC, pp. 990-993, 2010.

[39] A. Antonini, M. Butturi, P. Di Benedetto, E. Milan, D. Uderzo, P. Zurru, A. Parretta, N. Baggio, "Rondine PV concentrators: field results and innovations", Proc of $24^{\text {th }} \mathrm{EU}$ PVSEC, European Commission JRC, Pub. N. JRC54285, pp. 150-155, 2009.

[40] A. Antonini, M. Butturi, P. Di Benedetto, D. Uderzo, P. Zurru, E. Milan, M. Stefancich, M. Armani, A. Parretta, N. Baggio, "Rondine ${ }^{\circledR}$ PV concentrators: Field results and developments”, Progress in Photovoltaics, 17, pp. 451-459, 2009.

[41] A. Antonini, M. Butturi, P. Di Benedetto, D. Uderzo, P. Zurru, E. Milan, M. Stefancich, M. Armani, A. Parretta, N. Baggio, "Development of "Rondine" concentrating PV module: Field results and progresses", Proc. of $23^{\text {rd }} \mathrm{EU}$ PVSEC, European Commission JRC, pp. 898-902, 2008.

[42] A. Antonini, M. Butturi, P. Di Benedetto, D. Uderzo, P. Zurru, E. Milan, M. Stefancich, M. Armani, A. Parretta, N. Baggio, "Experimental results of a new PV concentrator system for silicon solar cells", Proc. of $22^{\text {th }}$ EU PVSEC, European Commission JRC, pp. 727-730, 2007 